\title{
Motor and Visual Imagery as Two Complementary but Neurally Dissociable Mental Processes
}

\author{
A. Sirigu and J. R. Duhamel
}

\begin{abstract}
Recent studies indicate that covert mental activities, such as simulating a motor action and imagining the shape of an object, involve shared neural representations with actual motor performance and with visual perception, respectively. Here we investigate the performance, by normal individual and subjects with a selective impairment in either motor or visual imagery, of an imagery task involving a mental rotation. The task involved imagining a hand in a particular orientation in space and making a subsequent laterality judgement. A simple change in the phrasing of the imagery instructions (first-person
\end{abstract}

or third-person imagery) and in actual hand posture (holding the hands on the lap or in the back) had a strong impact on response time (RT) in normal subjects, and on response accuracy in brain-damaged subjects. The pattern of results indicates that the activation of covert motor and visual processes during mental imagery depends on both top-down and bottom-up factors, and highlights the distinct but complementary contribution of covert motor and visual processes during mental rotation.

\section{INTRODUCTION}

Form your mouth into the shape required to pronounce the sound of an " $\mathrm{O}$ " and try to imagine yourself uttering that of an "f." With this simple exercise, the nineteenth century Viennese psychologist Stricker (1885) described a fascinating property of mental processes. This observation led him to propose that since the covert simulation of an action can be so easily disturbed by the presence of incompatible postural signals, it probably involved processing resources which are also responsible for the performance of the action itself. It even suggested a method by which the characteristics of our mental representations can be investigated experimentally. Contemporary research has confirmed the striking parallelism that exists between simulated and executed actions: the time course of mentally simulated movements is highly correlated to their actual execution (Johnson, 2000a; Crammond, 1997; Sirigu et al., 1996; Jeannerod, 1994; Parsons, 1994; Decety \& Michel, 1989); vegetative responses associated with physical effort vary in the same manner during both motor imagery and motor performance (Decety et al., 1991); motor-evoked potentials are selectively facilitated during simulated movements (Fadiga et al., 1995); overlapping brain networks are activated by the same movement when it is simply imagined or when it is actually executed (Gerardin et al., 2000; Porro et al., 1996; Parsons et al., 1995; Decety et al., 1994). Thus, motor imagery might corre-

CNRS, Lyon, France

spond to a process by which the brain activates a motor plan and monitors its unfolding through internal feedback signals, while maintaining motoneuronal output in a state of tonic inhibition or subliminal activation.

Mental imagery is obviously not limited to covertly performed motor actions. Following up on Stricker's ideas, the French psychologist Binet (1886), argued for the modularity of mental imagery functions, suggesting that just as imagining a movement draws on motor processing resources, imagining a form or a color draws on visual processing resources. As in the motor domain, there is substantial evidence for an area of overlap between the neural mechanism involved in visual imagery and in visual perception (for reviews, see Farah, 2000; Kosslyn \& Thompson, 2000; Goldenberg, 1993). Neuropsychological studies suggest that different brain lesions can lead to distinct forms of mental imagery impairments. In a previous study, we reported that the patients with damage to the posterior parietal cortex are poor at motor imagery (Sirigu et al., 1996). For instance, if normal subjects are asked to simulate a finger opposition sequence to the increasingly rapid beat of a metronome, they can estimate with remarkable accuracy the breakpoint in actual performance. Motor-impaired patients with lesions in the descending cortical pathway (Johnson, 2000b; Sirigu et al., 1995) or with Parkinson's disease (Dominey, 1995) also simulate limb movements accurately. By contrast, patients with lesions restricted to the parietal lobe show a very weak correlation between imagined and executed movement speed, indicating that the parietal cortex plays an important role 
in monitoring the internal signals generated by mental rehearsal. Evidence exists for impairments of visual imagery, which are generally associated with lesions in the associative visual pathways, and are characterized by an inability to generate visual images or experience visual dreams (Goldenberg, 1989, 1992; Farah, Levine, \& Calvanio, 1988).

A question that remains largely open, however, is that of the functional relations between motor and visual imagery. For instance, it has been proposed that cognitive tasks, such as mental rotation, might involve simulated motor acts and their anticipated visual results (Wexler, Kosslyn, \& Berthoz, 1998; Wohlschlager \& Wohlschlager, 1998). In sports psychology, motor rehearsal is often referred to as a method to "visualize" oneself in action, suggesting that the mental simulation of an action necessarily involves both covert visual and motor components. To what extent is this the case, and how separable are the underlying functional and cere- bral mechanisms? The present study addresses this issue by asking whether the same cognitive operation can be performed using motor or visual imagery, and whether the use of each of the two imagery modalities can be selectively facilitated or blocked through experimental manipulations or as a result of damage to key cerebral structures.

Here, we investigate the characteristics of a mental rotation in normal human subjects who were instructed to covertly evoke the image of a hand in a specific spatial orientation and to respond to queries about the location of a single finger on the imagined hand (Figure 1). There are, in principle, at least two ways in which such an image can be generated: by internally simulating a movement of one's hand which brings it into the requested position, hence using motor imagery, or by treating the hand not as one's own body part but as an external object whose orientation in space can be evoked from long-term memory without simulating an
Figure 1. (A) Imagery instructions for the hand rotation task. The same instruction could be phrased to generate a mental image in the first person or third person. (B) Actual hand postures imposed to the subjects while they were listening to the imagery instructions.

\section{A. Imagery instructions}

First person :

a Imagine yourself looking at the back of YOUR left hand, fingers pointing down. "
Third person : \& Imagine yourseif looking at the back of MY left hand, fingers pointing down. "

\section{B. Hand posture}

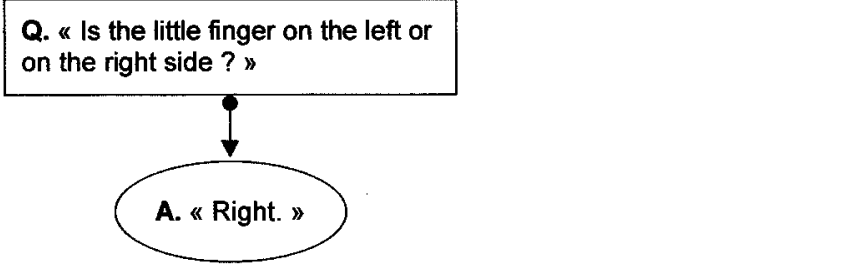

Hands on lap

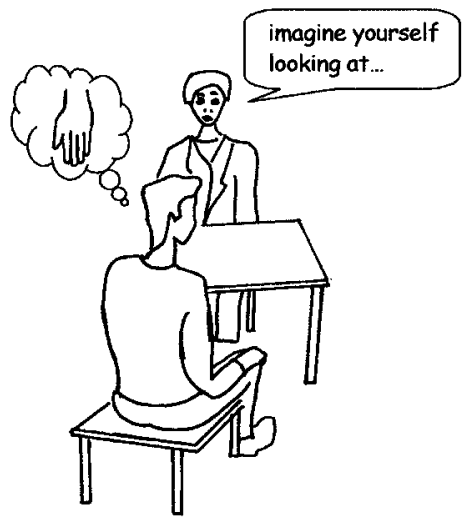

Hands in the back

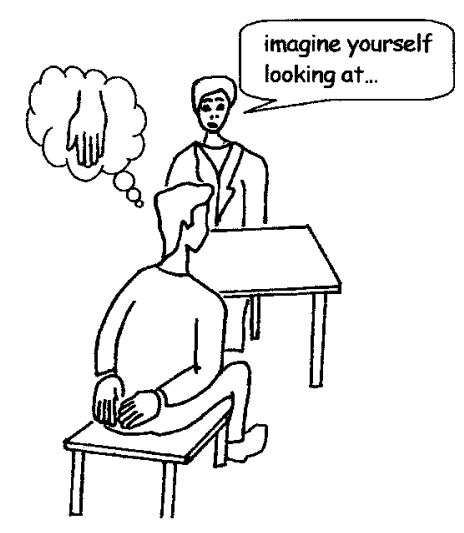


actual motor action. It should be kept in mind that there are no visual stimuli in the task (subjects' eyes are closed) but only spoken queries. In an attempt to orient subjects toward one or the other strategy, we introduced specific task constraints. The first was requiring subjects to imagine their own hand ("first-person" imagery) or another person's hand ("third-person" imagery). It should be noted that the correct answer to the query is the same in both cases: The difference lies in the manner in which the mental image of the hand is being generated, and not in the end result. The second constraint was requiring subjects to keep their hands on their lap or behind their back. While it is possible that both motor and nonmotor processes might be involved in this task, we hypothesized that thinking about one's own hand will primarily induce a covert motor simulation process and that mental imagery in this condition will be sensitive to variations in actual hand posture. Thinking about another person's hand will activate visual rather than motor processes and consequently be less sensitive to hand posture variations.

We also investigate this issue in subjects showing selective impairments in motor or visual imagery as a result of brain damage. Two patients with contrasting patterns of performance on tasks of motor and visual imagery were tested on the same mental rotation task used in normal subjects. We hypothesized that both patients could adequately generate a mental image of a hand under task conditions that call for their unimpaired imagery mode, but show deficits under conditions that call for the impaired imagery mode.

\section{RESULTS}

\section{Motor and Visual Imagery Contribute to Mental Rotation in Normal Subjects}

For the sake of simplicity, the results for "lap" and "back" hand postures are presented successively. As error rates were low under all conditions, we restricted our analyses of normal subjects' performances to response latencies (Figure 2). Since no significant effects of laterality of imagined hand or imagined hand orientation were found, these factors were not analyzed further.

\section{Hands on the Lap Condition}

The motor simulation hypothesis predicts that a relative advantage would be obtained by thinking about one's own hand, as opposed to thinking about another person's hand. This is what we found in the condition in which subjects rested their hands on their lap. Response time (RT) was more than three times as fast under firstthan third-person imagery (means 1.5 and $5.1 \mathrm{sec}$, respectively, $p<.01$ ). This effect probably results from the fact that first-person imagery allows a very direct mapping of the suggested mental image onto one's own

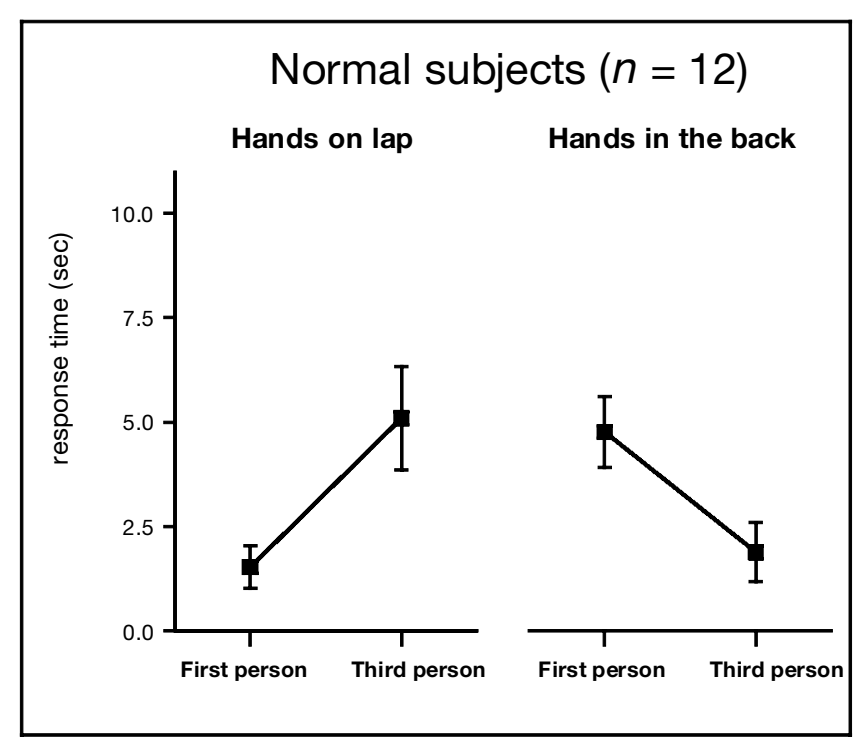

Figure 2. RT of normal subjects in the hand rotation task as a function of instruction type and hand posture (error bars = standard deviation).

body schema. It is crucial to always bear in mind that the only difference between the imagery instructions under first- and third-person conditions is the substitution of the word "my" by "your," and that the response to the query is the same in both conditions. Yet this simple manipulation is sufficient to modify radically the amount of time required to form a mental image and reach a decision.

\section{Hands in the Back Condition}

On the basis of Stricker's early observations, we expected that adopting a hand posture, which is incompatible with the suggested hand rotation, would interfere with motor simulation. Comparison of Figure $2 \mathrm{~A}$ and $\mathrm{B}$ for first-person instructions confirms the strong detrimental effect of an incompatible hand posture on response latency. Surprisingly, hand posture influences performance under third-person instructions as well but in the opposite direction, with "faster" responses with hands in the back than on the lap $(p<.01)$, RTs being an almost exact mirror image of those observed under first-person instructions.

Such a strong interaction between instruction type and hand posture suggests that two different cognitive processes are at play under first- and third-person imagery instructions, and that each operate under different constraints. In a laterality judgement task for visually presented hand shapes, Parsons (1994) showed that RT is longer by about $200 \mathrm{msec}$ when the subjects' own hands are outstretched forward as opposed to resting on the lap. This effect was interpreted in terms of differences in the length of the imagined motor path from the subject's current posture into the posture of the visual stimulus in the two conditions. A similar line of reasoning could, in principle, be applied to the effects 
of hand posture in first-person imagery. However, the magnitude of the difference ( $>3 \mathrm{sec}$ ) and the phenomenal experience reported by the subjects suggest an alternative interpretation. With the hands resting on the lap, the subjects reported that performing the task felt relatively natural, but with the hands held in the back, the whole process of imagining their hand in front of their line of gaze became very effortful. This suggests that keeping the hands behind the back does more than merely increase the length of the path from a current to an imagined hand position, and may actively interfere with the subject's attempt to simulate a rotation of their own hand. While introspection is not demonstration, it does seem to correlate quite well with RT. For instance, under third-person imagery, subjects described the opposite phenomenon. Those who had been first tested with their hand on the lap typically reported a subjective sense of facilitation when they held their hands behind, "out of the way," as though this posture made it easier for them to form a representation of the experimenter's hand as dissociated from their own.

Thus far, our results are consistent with the hypothesis that mentally creating an image of one's own hand in a particular orientation engages motor processes that simulate an actual hand rotation. This hypothesis also predicted that subjects would be faster overall for firstperson than third-person imagery. However, the results show that this is true only with the hands on the lap, and that subjects are better at third-person than first-person imagery with their hands behind the back. This has two implications which lead us to revise our initial working hypothesis. First, the fact that third-person imagery is not immune to hand posture manipulations suggests that some degree of motor processing is present even in this condition. Covert motor activation may automatically occur in any imagery task which involves a moving body part, or perhaps whenever some kind of spatial transformation must be applied to the content of the mental representation. Second, assuming, on the basis of first-person imagery results, that motor activation is enhanced with a hands forward posture and inhibited with a hands in the back posture, the faster RT in the latter condition for third-person imagery implies that imagining a hand construed as somebody else's is best achieved through a nonmotor route. We propose that this alternative imagery mode primarily involves that manipulation of visual rather than motor images, and we put this idea to test in a patient with visual imagery deficit and inferotemporal lobe damage (J.B.) and in a patient with a motor imagery deficit and left parietal lobe damage (R.L.).

\section{Motor and Visual Imagery Impairments are Functionally Dissociable}

An essential preliminary step is to establish that impairments in visual and motor imagery occur selectively by showing that patients with lesions in different brain regions show completely opposite patterns of performance on two independent sets of mental imagery tasks. One patient, R.L., had a left parietal lobe tumor, with limb apraxia and motor imagery impairment. The second patient, J.B., had bilateral damage to the inferotemporal structures, and at the time of testing showed no visual recognition impairments but a persistent incapacity to mentally visualize the shape of objects and faces.

\section{Motor Imagery}

Two tests of manual motor imagery were used (Figure 3). The first involves simulated and executed pointing hand movements to different-sized visual targets. In normal subjects, movement duration in both conditions is highly correlated and shows a strict dependency on target size. By contrast, the parietal patient, R.L., showed a complete lack of correlation between executed and imagined movements. Trying to imagine his hand in motion required a great mental effort, and he often reported "losing" the image of his hand the moment he mentally set it in motion. The second task involves mentally rehearsing a finger opposition sequence to the increasing pace of a metronome. The maximum subjective speed achieved is later compared to the actual breakpoint when the same procedure was actually performed. Patient R.L. largely underestimated his actual motor performance. By contrast, the inferotemporal patient J.B. showed a normal correlation between simulated and executed movement speeds in both tasks. Interestingly, this subject expressed surprise at how easy it was to imagine himself executing an action, reporting compelling kinesthetic sensations despite the fact that the only visual image of his hand he experienced was "a kind of stick."

\section{Visual Imagery}

Visual imagery capacities were assessed using several tasks (Farah, 2000). On tests requiring to report on visual attributes of named objects and animals, the parietal patient R.L. obtained near-perfect scores, while the inferotemporal patient J.B. was severely impaired (Table 1). For instance, he could not identify the color of named objects although he could recognize the same objects and name their color when presented visually. He was unable to categorize named animals by a shape attribute, this despite the fact that he adequately categorized them according to habitat. J.B. scored at chance on a mental rotation task adapted from Shepard and Metzler (1971) in which an abstract drawing must be matched to the same drawing presented in a different orientation among distractors. This patient thus appears to show deficits both in accessing stored visual information and in the on-line manipulation of visual shapes in a 
Figure 3. Motor imagery tasks. (A) Mentally simulated pointing movement to different-sized visual targets by the two patients. Symbols represent three nonconsecutive replications of the same trial type, and the dashed and solid lines correspond to the nonlinear regression fit applied to individual trial data for imagined and executed movements, using the equation: $f=a+b\left(\log _{2}(2 A / W)\right)$, where $A$ and $W$ represent movement amplitude and target width, respectively. In this version of the task, $A$ was a constant. The graph only shows the data for the left hand of each patient. Similar results were obtained for the right hand. For patient J.B., correlation coefficients between imagined and executed movement was .81 and .84 for the right and left hands, respectively, a performance comparable to that of normal subjects. For patient R.L., correlation coefficients between imagined and executed movement were -.20 and .34 for the left and right hands, respectively. (B) Speeded thumb-finger opposition sequence in the two patients. Subjects first simulated the finger sequence to the increasing pace of a metronome. Individual points for each patient plot predicted (imagined) against actual performance breakpoint for two trials each with the left and right hands. In this task, normal subjects' performance clusters along the $45^{\circ}$ line, and errors in predicting actual motor performance by mental simulation do not exceed $8 \%$ (data not shown). R.L.'s performances in these two motor imagery tasks were included in a somewhat different format in a prior study on a series of brain-damaged patients (Sirigu et al., 1996).

\section{A. Visuo-manual pointing}
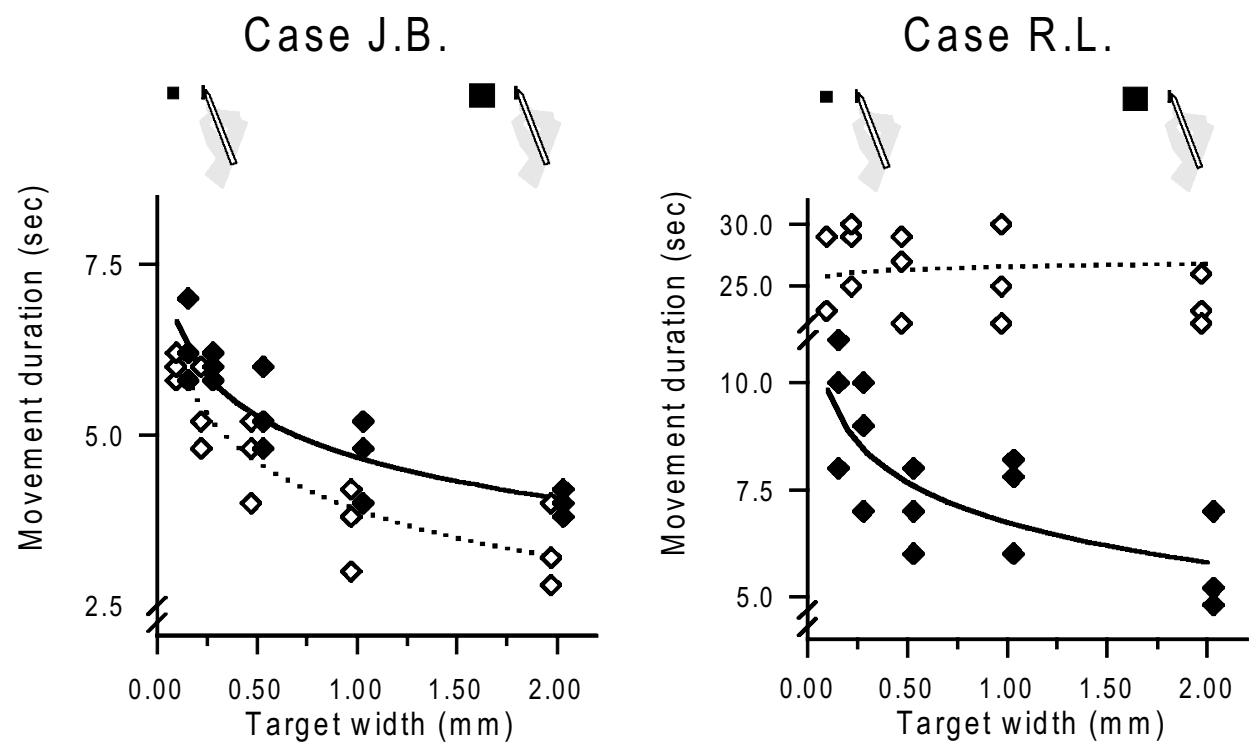

\section{B. Finger opposition sequence}
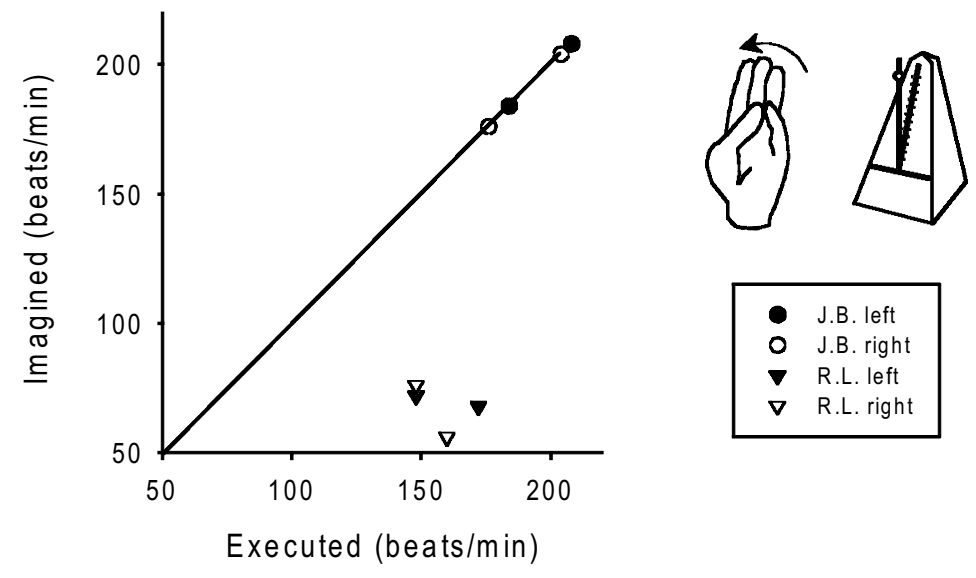

- J.B. left

O J.B.right

$\nabla$ R.L. left

$\nabla$ R.L. right

\section{8}




\begin{tabular}{|c|c|c|c|}
\hline & $\begin{array}{c}\text { Case J.B. } \\
\text { (inferotemporal lesion) }\end{array}$ & $\begin{array}{c}\text { Case R.L. } \\
\text { (parietal lesion) }\end{array}$ & $\begin{array}{l}\text { Normals } \\
(n=12)\end{array}$ \\
\hline 1. Identify color of a named object & $8 / 20^{*}$ & $19 / 20$ & $18.5 / 20$ \\
\hline 2a. Categorize named object by shape & $7 / 20 *$ & $20 / 20$ & $19 / 20$ \\
\hline 2b. Categorize named object by semantic attribute & $20 / 20$ & $20 / 20$ & $19.3 / 20$ \\
\hline
\end{tabular}

Performance of the two patients and normal controls on tasks of visual imagery for colors and shapes, and of mental rotation of abstract shapes. Task $2 \mathrm{~b}$ serves as a control for the integrity of knowledge of non-visual properties of the items used in Task 2a. Results marked with * indicate a score more than 3 standard deviations below normal subjects.

response latencies and error rates (i.e., reporting the wrong side for the queried finger location) were of interest.

Several points can be noted regarding the performance of the inferotemporal patient J.B. (Figure 4). As in normal subjects, he responded slower in the thirdperson compared to the first-person imagery condition while holding his hands on the lap $(p<.01)$. However, with the hands in the back, RTs are also slower for thirdperson than first-person instructions, and thus do not show the reversal observed in normal subjects. Importantly, J.B. made a large numbers of errors in almost all conditions, with the notable exception of the first-person/lap posture combination, in which he scored perfectly. It should be noted that the error rates are slightly higher than would be expected by chance, that is, the patient showed a certain systematic tendency to answer "right" when the correct answer was "left" or vice-versa, which might imply that a correct hand representation of the hand was evoked at some level on a proportion of error trials. However, this trend was not statistically significant in any of the conditions ( $p>.29$ or more). The general pattern of results strongly suggests that J.B. mainly relied on covert motor processes to perform the hand rotation task. The fact that performance under first-person imagery deteriorated when the hands were placed behind the back indicates that adopting an incompatible hand posture does not simply increase the subjective distance between the actual and imagined hand position, but that it interferes with the covert motor simulation process. Finally, these results support the hypothesis that intact visual imagery mechanisms are involved in this task, and critically so when a simulated rotation of one's own hand cannot be achieved.

In sharp contrast to his marked impairment on motor imagery tasks, the parietal patient, R.L., made relatively few errors on the hand rotation task, but two interesting trends can be observed with respect to response latencies (Figure 5). First, under "lap" hand posture condition, no RT facilitation was observed when asked to imagine his own hand: R.L. employed the same amount of time for first-person and third-person instructions. Introspecting about his performance, R.L. stated that he never imagined moving his hand, but that he instead imagined directly the suggested posture at its final position. Second, contrary to patient J.B., but just like normal subjects, R.L. shows facilitation under thirdperson instructions when holding the hand behind his back, suggesting some benefits from conditions which promote a visual imagery mode.

\section{DISCUSSION}

The present results show that forming a mental representation of one's own hand activates motor representations. In normal subjects, changing the actual posture of the hand affects the ease with which this mental representation is generated. A standard hand posture, such as resting the hands on one's lap, facilitates the process of mentally rotating the hand in a given position, while a more awkward posture, such as holding the hands behind the back, has the opposite effect. Postural signals thus appear to interact with mental representations in a bottom-up manner, a phenomenon analogous to the one described by Stricker (1885) regarding the effect of congruent and incongruent mouth postures on the mental rehearsal of phonemes, and more recently by Parsons (1994) in the context of a handedness decision task. The present results show a further aspect of this interaction wherein the dependency on hand posture is mirror-reversed when imagining another person's hands. This indicates that quite different mechanisms mediate imagery in the first person and in the third person, and leads to our second conclusion that topdown cognitive processes play a major role in the selection of processing resources during mental manipulation tasks: While first-person imagery might naturally, or even obligatorily, involve motor activation, thirdperson imagery might operate using preferentially nonmotor mechanisms.

\section{Covert Motor Processing and Mental Imagery Performance}

What is the origin of the observed effects of hand posture? These effects are not mediated by visual cues 
Case J.B. (infero-temporal lesion)

A. Response time

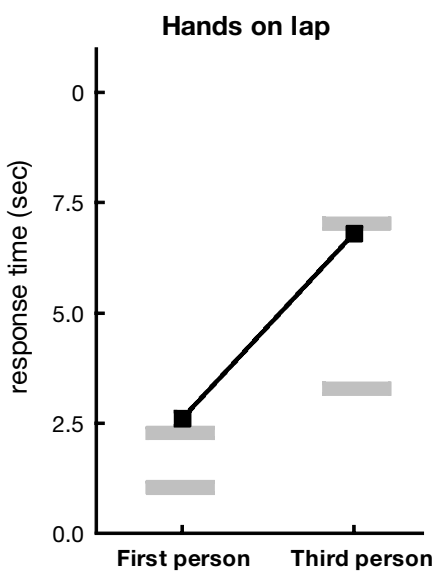

Hands in the back
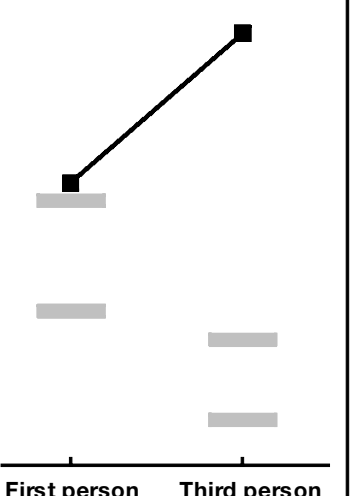

B. Number of errors

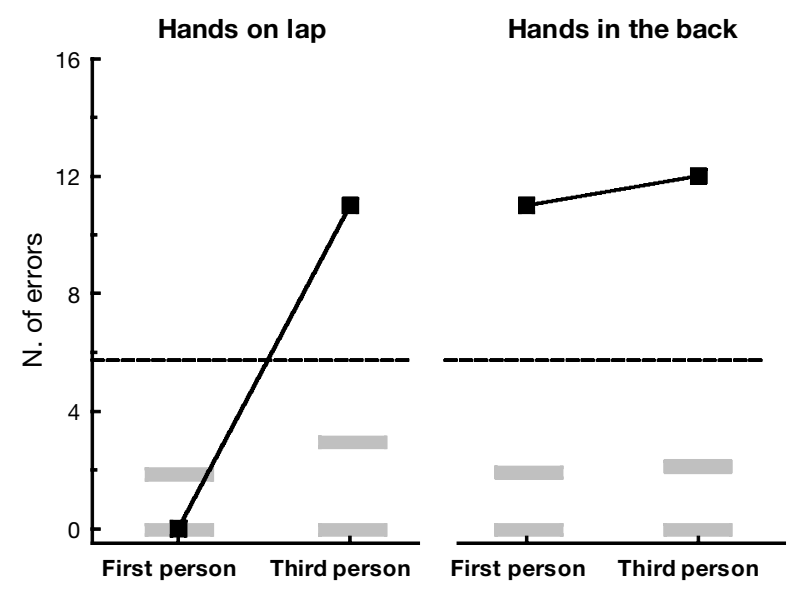

Figure 4. (A) RT and (B) number of errors for the inferotemporal patient J.B. on the hand rotation task. Curves represent the patient's data and the horizontal gray bars represent the lower and upper bounds of normal subjects' performance. The horizontal dashed line in the bottom graph shows chance level.

since the subject keeps their eyes closed throughout the task. The facilitation obtained under first-person imagery could perhaps result from an attentional effect related to the greater saliency of the more "canonical" of the two postures. However, this cannot explain the fact that facilitation was reversed in favor of the less canonical posture under third-person imagery. A more plausible possibility in our view is a form of subliminal motor activation, resulting from maintaining the hands forward, in a "ready-for-action" position. In the context of first-person imagery, which affords a direct mapping of the imagined hand onto one's own body representation, this would offer a definite processing advantage. By contrast, under third-person imagery, such motor facilitation would produce interference through the simulta- neous activation of competing hand representations: that of the examiner's, dictated by the instructions, and that of the subject's, mandatorily activated by the hands forward posture. The effects of holding the hands behind the back, which facilitates third-person imagery but impairs first-person imagery, are seemingly paradoxical. Two interpretations, which are not mutually exclusive, can be advanced. The first is that actively holding the hands in the back puts some kind of mental "handcuffs" on motor imagery, impeding first-person imagery and freeing third-person imagery from conflicting interference. The second is that the space behind the back has a distinct status. For instance, Parsons and Shimojo (1987) showed that hand posture influences the interpretation of haptic letter shapes: For the hand forward palm down, letters drawn on the back are perceived normal, and those drawn on the palm are mirror-reversed, suggesting that the subject mentally

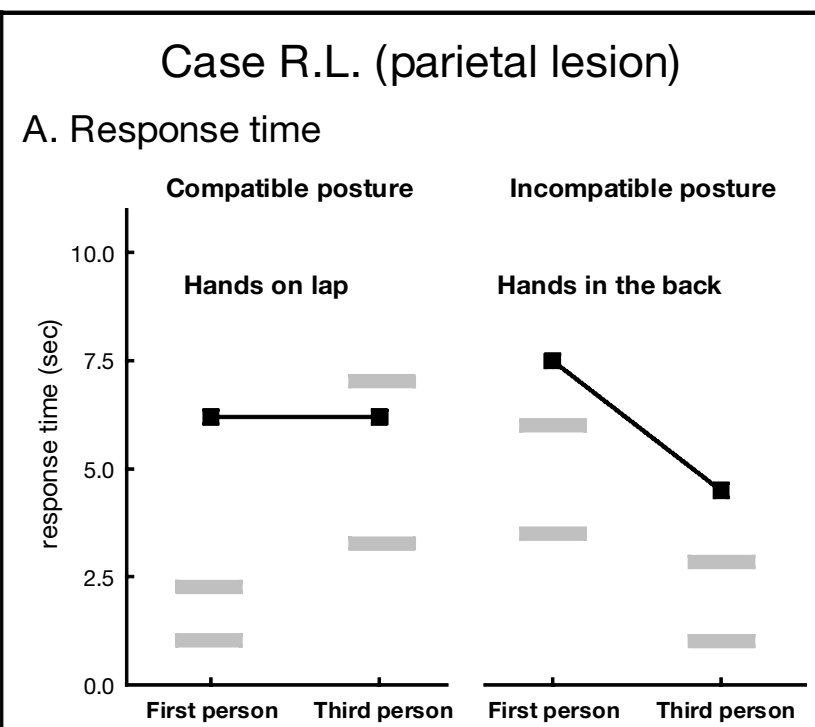

\section{B. Number of errors}

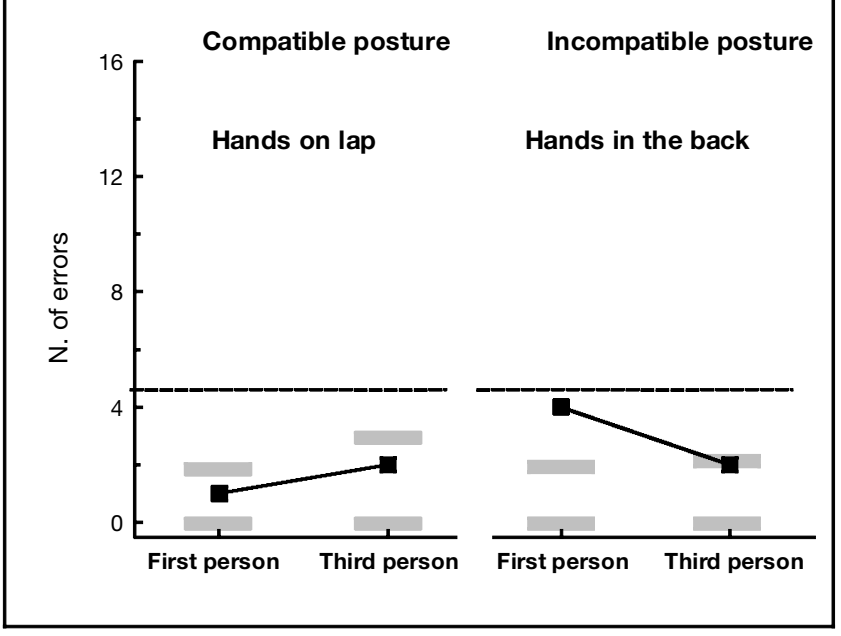

Figure 5. (A) RT and (B) number of errors for the left parietal patient R.L. on the hand rotation task. Conventions as in Figure 4. 
rotates his hand to "see" the letter traced on his palm before interpreting it. With the hand behind the back, letters on either palm or back are interpreted in the same way, as though the letter shape was registered with respect to an external reference, independent of hand orientation. It is thus possible that adopting a thirdperson viewpoint to assess a left-right finger pattern might be facilitated when the subject's own hands are registered relative to a nonegocentric frame in back space.

Activation of covert motor processes during mental rotation has been proposed in previous studies. Although first-person imagery was never explicitly required in these investigations, the nature of the stimuli that were used likely promoted this strategy, at least implicitly. In laterality judgements for pictures of left and right hands, Parsons (1994) reported that decision times of normal subjects depend not so much on the actual angle of rotation of the hand, as on specific joint constraints imposed on possible (simulated) motor paths. This suggests that subjects formed their decision after having mentally simulated a movement of their own hand, in order to match the position of the test stimulus. We did not observe such systematic effects of hand orientation on RT, but this is probably due to the relatively low temporal resolution of our experimental paradigm. The influence of hand orientation reported by Parsons is the 100-300-msec range, while with auditorily presented imagery instructions, RT differences inferior to $1 \mathrm{sec}$ cannot be realistically detected. Another study by Johnson (2000a) also showed that anticipated biomechanical constraints are applied to awkwardness judgments made before grasping oriented objects. Consistent with such behavioral data, brain imaging with PET showed that performance of a mental rotation using hands as visual stimuli recruits an extensive cortical network that includes the visual areas, parietal lobe areas, and motor and premotor structures in the frontal lobe (Kosslyn et al., 1998). These authors report that the same type of mental rotation applied to abstract drawings recruits mostly posterior visual cortical structures. Other studies have also demonstrated the involvement of the motor system during mental rotation (Ganis et al., 2000; Cohen et al., 1996).

The case for the contribution of motor processes to mental imagery can be extended with our observations made in brain-damaged subjects. The fact that patient J.B., who lacked visual imagery abilities, was as good as normal subjects at anticipating motor performance during simulated finger sequences or manual pointing is remarkable in itself. These explicit motor imagery tasks were reported by the patient to produce sharp movement sensations devoid of any visual content. In the hand rotation task, the patient relied entirely upon motor imagery. Localizing fingers on an imaginary hand was possible in the first person but not in the third person. A further demonstration of the contribution of motor processes to cognitive performance was found when comparing letters displayed in upper case print on the basis of their imagined lower case appearance. Unless micromovements of the fingers were allowed, patient J.B. performed essentially at chance. Thus, information about letter shapes could be extracted indirectly via kinesthesia, e.g., from the motor paths of the fingers in the simulated act of writing, but a direct route to a visual store of letter forms was not accessible.

\section{Relative Contribution of Motor and Visual Imagery to Mental Rotation}

The latter observation suggests that motor processing can contribute to mental manipulation of objects that are not body parts, but which can nevertheless be acted upon. It has been proposed that mental rotation might involve simulating motor actions such as manually grasping one of the objects and aligning its main axis with that of the other object. Wexler et al. (1998) showed that during mental rotation of abstract shapes, an accompanying unseen hand movement by the subject made in a direction compatible with the mental rotation produces faster performance than an incompatible movement. Our results suggest that such a coupling between visual and motor processes during mental rotation might depend on the parietal lobe, which we have previously shown to be a critical site for the activation of covert motor processes during simulated motor acts (Sirigu et al., 1996). In the present study, the patient with parietal lesion was reasonably accurate on the hand rotation task overall, but when imagining his own hand, he did not show the RT benefit for the compatible hand posture seen in all normal subjects and in the patient with temporal lobe lesions. This suggests that intact visual imagery may be a sufficient condition for mental rotation performance, but in cases of parietal damage, visual and motor imagery mechanisms cannot be coupled.

To conclude, a cognitive task such as mental rotation of imagined hands is accomplished by calling upon different subsets of processing resources, which, at a functional level, correspond to different routes toward a single solution. Under instructions to seek this solution using imagery in the first person, subjects use primarily motor resources, and under instructions to seek the solution using imagery in the third person, they use primarily visual resources. Our data in brain-damaged subjects further support this claim. Lesions causing visual imagery impairments selectively preserve mental rotation guided by motor imagery, while lesions causing motor imagery impairments selectively preserve performance guided by visual imagery. These results are consistent with a view of mental imagery, which allows a close coupling of covert motor and visual processes, which in turn depend upon modularly organized neural mechanisms. 


\section{METHODS}

\section{Subjects}

Two patients (J.B., R.L.) with focal cortical lesions and 12 age- and education-matched normal controls (NCs) (mean age $=40.2$ years, $S D=9.0$ years) participated in this study. Patients' informed consent was obtained prior to testing. Patient J.B. (55 years old) sustained herpes encephalitis 5 years before the study. The initial neuropsychological examination showed prosopagnosia and object agnosia. The patient also complained of a total incapacity to visualize the shape or color of familiar objects and faces. An MRI scan revealed lesions in both temporal poles. At the time of this study, visual recognition of objects, colors, and faces had returned to normal. However, the patient still reported an inability to "form pictures in his head." Patient R.L. (29 years old) had a parietal astrocytoma in the left angular and supramarginal gyri. At the time of the study, he presented with a bilateral ideomotor apraxia and showed difficulties in manipulating objects without visual guidance. There were no visuospatial or attentional impairments, or elementary motor or sensory deficits, body schema disorders, right-left confusion, or asomatognosia.

\section{Motor Imagery Tasks}

Two tasks were used (Sirigu et al., 1996). In the first, subjects kept the tip of a stylus stationary at a starting position and mentally pointed to a visual target $1.25,2.5$, 5,10 , or $20 \mathrm{~mm}$ wide, at distance of $30 \mathrm{~mm}$. Subjects started at a go signal and simulated moving back and forth five consecutive times between the starting and target locations. Mental movement duration was the time elapsed between the go signal and verbal report of sequence completion, and was compared to actual movement duration for the same sequences. Imagined and executed movements were conducted in blocks, and motor imagery trials were always completed before actual execution. In normals, imagined and actual movement time are correlated, and are inversely related to target width, consistent with Fitts' (1954) Law. The second task measured the capacity to predict the maximum speed, which can be achieved during a continuous sequential thumb-finger opposition movement. A metronome set the pace, starting at 60 beats per min and increasing progressively in 10 beats steps. Subjects first mentally simulated the sequence and reported when they were no longer able to keep up with the metronome speed. They were later tested during actual execution.

\section{Visual Imagery Tasks}

In the first task, subjects were asked to report the color of named objects belonging to living or nonliving categories (What is the color of a tire? of a ripe cherry?). A second task tested the mental representation of form by asking subjects to judge the relative size of an animal's tail (Does a pig, a cat ... have a long or short tail in proportion to its body?). As a control, judgments were also made about nonvisual semantic attributes of the same stimuli (Is France a natural habitat for a lion, a pig ... ?). A third task evaluated mental rotation of twodimensional geometrical shapes by matching a test stimulus to one of three other stimuli rotated $45^{\circ}$, $90^{\circ}$, or $135^{\circ}$, only one of which had the same shape as the test stimulus. Normal subjects scored over $90 \%$ correct on all three visual tasks.

\section{Hand Rotation Task}

The task involved listening to verbal descriptions of a hand posture. All different possible combinations of hand ownership, laterality were tested (own/examiner's hand, left/right hand, fingers pointing up/down, facing palm/back). This was followed by a query about the location of either the thumb or the little finger, thus yielding 16 possible instruction/query combinations for first- and third-person instructions. Experimental sessions alternated eight-trial blocks of first- and thirdperson imagery conditions, which were counterbalanced across subjects. Each subject was tested twice. For half of the subjects, the first session was conducted with hands on the lap palms facing down and the second one with hands in the back palms facing outward, and for the other half, the session order was reversed. Subjects were seated across a desk, facing the examiner with their eyes closed throughout the blocks of trials. They were told to imagine themselves looking at a hand located directly in front of their face, and to make the decision about finger location from their own viewpoint. Pilot trials were run to ensure that instructions were understood. Data on RT and errors were analyzed with repeated-measures ANOVA and a posteriori pairwise comparisons.

Reprints requests should be sent to Dr. Angela Sirigu, Institut des Sciences Cognitives, CNRS, 67, BD Pinel, 69675 Bron, France, or via e-mail: sirigu@isc.cnrs.fr.

\section{REFERENCES}

Binet, A. (1886). La Psychologie du Raisonnement. In Alcan (Ed.). Paris.

Cohen, M. S., Kosslyn, S. M., Breiter, H. C., DiGirolamo, G. J., Thompson, W. L., Anderson, A. K., Brookheimer, S. Y., Rosen, B. R., \& Belliveau, J. W. (1996). Changes in cortical activity during mental rotation. A mapping study using functional MRI. Brain, 119, 89-100.

Crammond, D. (1997). Motor imagery: Never in your wildest dream. Trends in Neuroscience, 20, 54-57.

Decety, J., \& Michel, F. (1989). Comparative analysis of actual and mental movement times in two graphic tasks. Brain and Cognition, 11, 87-97.

Decety, J., Jeanneorod, M., Germain, M., \& Pastene, J. (1991). Vegetative response during imagined movement is proportional to mental effort. Behavioural Brain Research, 42, $1-5$. 
Decety, J., Perani, D., Jeannerod, M., Bettinardi, V., Tadary, B., Woods, R., Mazziotta, J. C., \& Fazio, F. (1994). Mapping motor representations with PET. Nature, 371, 600-602.

Dominey, P., Decety, J., Broussolle, E., Chazot, G., \& Jeannerod, M. (1995). Motor imagery of a lateralized sequential task is asymmetrically slowed in hemi-Parkinson's patients. Neuropsychologia, 33, 727-741.

Fadiga, L., Fogassi, L., Pavesi, G., \& Rizzolatti, G. (1995). Motor facilitation during action observation. A magnetic stimulation study. Journal of Neurophysiology, 73, 2608-2611.

Farah, M. J. (1989). The neural basis of mental imagery. Trends in Neuroscience, 12, 395-399.

Farah, M. J. (2000). The neural basis of mental imagery. In M. Gazzaniga (Ed.), The new cognitive neuroscience (pp. 965974). MIT Press.

Farah, M., J., Levine, D. N., \& Calvanio, R. A. (1988). A case study of mental imagery deficit. Brain and Cognition, 8, 147-164.

Fitts, P. M. (1954). The information capacity of the human motor system in controlling the amplitude of movement. Journal of Experimental Psychology, 47, 381.

Ganis, G., Keenan J. P., Kosslyn, S. M., \& Pascual-Leone, A. (2000). Transcranial magnetic stimulation of primary motor cortex affects mental rotation. Cerebral Cortex, 10, 175-180.

Gerardin, F., et al. (2000). Partially overlapping neural networks for real and imagined hand movements. Cerebral Cortex, 10, 1093-1101.

Goldenberg, G. (1989). The ability of patients with brain damage to generate mental visual images. Brain, 112, 305-325.

Goldenberg, G. (1992). Loss of visual imagery and loss of visual knowledge-a case study. Neuropsychologia, 30, 1081-1099.

Goldenberg, G. (1993). The neural basis of mental imagery. Baillieres Clinical Neurology, 2, 265-286.

Jeannerod, M. (1994). The representing brain. Neural correlates of motor intention and imagery. Behavioral and Brain Sciences, 17, 187-245.

Johnson, S. H. (2000a). Thinking ahead: The case for motor imagery in prospective judgements of prehension. Cognition, 74, 33-70.

Johnson, S. H. (2000b). Imagining the impossible: Intact motor representations in hemiplegics. NeuroReport, 11, 729-732.

Kosslyn, S. M., \& Thompson, W. (2000). Shared mechanisms in visual imagery and visual perception: Insights from cognitive neuroscience. In M. Gazzaniga (Ed.), The new cognitive neuroscience (pp. 975-985). MIT Press.

Kosslyn, S. M., DiGirolamo, G. J., Thompson, W. L., \& Alpert, N. M. (1998). Mental rotation of objects versus hands: Neural mechanisms revealed by positron emission tomography. Psychophysiology, 35, 151-161.

Kosslyn, S. M., Flynn, R. A., Amsterdam, J. B., \& Wang, G. (1990). Components of high-level vision: A cognitive neuroscience analysis and accounts of neurological syndromes. Cognition, 34, 203-277.

Parsons, L. (1994). Temporal and kinematic properties of motor behavior reflected in mentally simulated action. Journal of Experimental Psychology: Human Perception and Performance, 20, 709-730.

Parsons, L. M., \& Shimojo, S. (1987). Perceived spatial organization of cutaneous patterns on surfaces of the human body in various positions. Journal of Experimental Psychology: Human Perception and Performance, 13, 488-504.

Parsons, L. M., Fox, P. T., Downs, J. H., Glass, T., Hirsch, T. B., Martin, C. C., Jerabek, P. A., \& Lancaster, J. L. (1995). Use of implicit motor imagery for visual shape discrimination as revealed by PET. Nature, 375, 54-58.

Porro, C. A., Francescato, M. P., Cettolo, V., Diamond, M. E., Baraldi, P., Zuiani, C., Bazzocchi, M., \& di Prampero, P. E. (1996). Primary motor and sensory cortex activation during motor performance and motor imagery: A functional magnetic resonance imaging study. Journal of Neuroscience, 16, 7688-7698.

Shepard, R. N., \& Metzler, J. (1971). Mental rotation of threedimensional objects. Science, 171, 701-703.

Sirigu, A., Cohen, L., Duhamel, J. R., Pillon, B., Dubois, B., Agid, Y., \& Pierrot-Deseilligny, C. (1995). Congruent unilateral impairments for real and imagined hand movements. NeuroReport, 6, 997-1001.

Sirigu, A., Duhamel, J. R., Cohen, L., Pillon, B., Dubois, B., \& Agid, Y. (1996). The mental representation of hand movements after parietal cortex damage. Science, 273, 1564-1568.

Stricker, S. (1885). Du langage et de la musique. Paris, Biblioteque de Philosophie Contemporaine.

Wexler, M., Kosslyn, S. M., \& Berthoz, A. (1998). Motor processes in mental rotation. Cognition, 68, 77-94.

Wohlschlager, A., \& Wohlschlager, A. (1998). Mental and manual rotation. Journal of Experimental Psychology: Human Perception and Performance, 24, 397-412. 